\title{
Herpes zoster risk and burden of disease in immunocompromised populations: a population-based study using health system integrated databases, 2009-2014
}

Cintia Muñoz-Quiles $^{1 *+}$ (D) Mónica López-Lacort ${ }^{1 \dagger}$, Javier Díez-Domingo ${ }^{1,2}$ and Alejandro Orrico-Sánchez ${ }^{1}$

\begin{abstract}
Background: Estimate the incidence of herpes zoster (HZ), its complications and healthcare utilization rates in adults ( $\geq 18$-years-old) with a wide range of immunocompromised (IC) conditions compared to IC-free cohort.

Method: A population-based retrospective study using the Valencia healthcare Integrated Databases (VID) (20092014). $\mathrm{HZ}$ and IC were defined using ICD-9 codes in primary care (PC) and hospitalization registers. Incidence rates (IR), risk of $\mathrm{HZ}, \mathrm{HZ}$-recurrence, $\mathrm{HZ}$-complications and healthcare utilization rates were estimated in the IC-cohort compared to IC-free.

Results: The study population consisted of 4,382,590 subjects, of which 578,873 were IC (13\%). IR (in 1000 persons-year) of $\mathrm{HZ}$ overall, in $\mathrm{IC}$ and in IC-free cohort was 5.02, 9.15 and 4.65, respectively. IR of $\mathrm{HZ}$ increased with age in both cohorts and it was higher for all IC conditions studied, reaching up to twelvefold in subjects with stem cell transplantation. IC subjects had 51\% higher risk of developing HZ, 25\% higher HZrecurrence and the risk of $\mathrm{HZ}$-complications was 2.37 times higher than in IC-free. HZ-related healthcare utilization was higher in the IC-cohort than in IC-free (number of hospitalizations 2.93 times greater, hospital stays $12 \%$ longer, $66 \%$ more $\mathrm{HZ}$-specialist visits, $2 \%$ more PC visits, sick leaves $18 \%$ longer and $20 \%$ higher antiviral dispensation).

Conclusions: Patients suffering from all the IC conditions studied are at higher risk of developing $\mathrm{HZ}, \mathrm{HZ}-$ recurrence and post-herpetic complications, which implies a substantial morbidity and a high consumption of resources. These results should be considered for vaccine policy implementation.
\end{abstract}

Keywords: Herpes zoster, Immunocompromised, Epidemiology, Population-based study, Healthcare utilization, Vaccine

\footnotetext{
* Correspondence: cinquiles@gmail.com

${ }^{\dagger}$ Cintia Muñoz-Quiles and Mónica López-Lacort contributed equally to this work.

${ }^{1}$ Vaccines Research Unit, Fundación para el Fomento de la Investigación Sanitaria y Biomédica de la Comunitat Valenciana, FISABIO-Public Health, Avda. Cataluña, 21, 46020 Valencia, Spain

Full list of author information is available at the end of the article
}

(C) The Author(s). 2020 Open Access This article is licensed under a Creative Commons Attribution 4.0 International License, which permits use, sharing, adaptation, distribution and reproduction in any medium or format, as long as you give appropriate credit to the original author(s) and the source, provide a link to the Creative Commons licence, and indicate if changes were made. The images or other third party material in this article are included in the article's Creative Commons licence, unless indicated otherwise in a credit line to the material. If material is not included in the article's Creative Commons licence and your intended use is not permitted by statutory regulation or exceeds the permitted use, you will need to obtain permission directly from the copyright holder. To view a copy of this licence, visit http://creativecommons.org/licenses/by/4.0/ The Creative Commons Public Domain Dedication waiver (http://creativecommons.org/publicdomain/zero/1.0/) applies to the data made available in this article, unless otherwise stated in a credit line to the data. 


\section{Introduction}

One in three people aged between 50 and 90 years will develop herpes zoster ( $\mathrm{HZ}$ ) and approximately one in ten will develop postherpetic neuralgia (PHN, defined as dermatomal pain lasting for at least three months after the acute phase of a HZ) [1]. A decrease of the specific cell-mediated immunity against varicella zoster virus (VZV) seems to be the cause of HZ development as a consequence of the VZV reactivation [2]. It could explain the increased risk of suffering $\mathrm{HZ}$ in ageing people and especially in people with immunocompromised (IC) conditions due to diseases or treatments that alter the immune response (Immunodeficiency disorders and autoimmune diseases, human immunodeficiency virus (HIV), cancer, organ transplantation) [3-6].

Both, $\mathrm{HZ}$ and PHN result in reduced quality of life as well as individual and societal healthcare costs [6-8]. Its treatment, especially that of PHN, is extremely difficult and frustrating for both patients and specialists (who in most cases fail to mitigate the suffering of their patients) [9-11]. Considering these difficulties, a live attenuated vaccine (Zoster Vaccine Live, $\mathrm{ZVL}$ ) was commercialized in 2006 as a tool to prevent $\mathrm{HZ}$ and its complications, and it was licensed in many countries for its use in adults older than 50 years of age [12-14]. Despite its availability, there is relatively scarce use of the current live attenuated vaccine in Spain, where there are efforts to introduce more recommendations for $\mathrm{HZ}$ vaccination [15]. The identification and definition of priority $\mathrm{HZ}$ atrisk groups is a key step for the design of vaccination programs.

Previous studies on HZ and PHN epidemiology using Real World Data have been developed in order to identify highly susceptible populations such as patients with diabetes, chronic obstructive pulmonary disease (COPD) or heart failure (HF) among others [1, 4, 9, 16-18]. People with IC conditions are among the highest risk of developing $\mathrm{HZ}$ [3-5]. However these cohorts cannot benefit from ZVL vaccine due to its contraindication in IC populations [19]. This is the reason why these IC populations have been normally excluded from many epidemiological studies to avoid confusion $[1,16,17]$. As an alternative, an adjuvanted recombinant Zoster vaccine (RZV) has been developed and was first approved in Canada followed by the US in 2017 for its use in adults aged 50 years and older [20,21]. Clinical studies on immunogenicity, safety and efficacy of RZV have been completed or are ongoing for different IC conditions such as HIV, haematopoietic stem cell transplant (HSCT) or transplant recipients or Inflammatory bowel disease (IBD) [22-25]. Their results will allow broadening the approved indication of RZV [21].

The estimation of the full burden of $\mathrm{HZ}$ disease including $\mathrm{HZ}$ incidence and recurrence rates, its complications and the evaluation of $\mathrm{HZ}$ risk for specific IC subpopulations constitutes a critical step to make an estimation of future impact of the RZV in these risk groups. So far, only a few studies assessed HZ incidence among patients with different IC conditions including populations large enough to allow comparison among the different conditions [3-5, 26, 27]. However, none of them included risk estimations. As recently reviewed by Mareque et al., in Spain there is a lack of these types of studies and its heterogeneity and limited populations make it difficult to develop the definition of priority groups for the implementation of a future non-routine HZ vaccination program [15].

The Valencia Region of Spain has a population of around 5 million inhabitants, over $96 \%$ of which are insured by the Regional Health System (RHS) [1]. The Region counts with the Valencia health system Integrated Databases (VID), a healthcare electronic databases network gathering real world data (RWD) from hospitalization, primary care, specialist and medication, among other [28]. The quality of VID has been shown in several publications $[1,16,29-31]$. Using the VID, the objective of the present study was to estimate the risk of $\mathrm{HZ}$, its recurrence and complications in adults with a wide range of IC conditions in comparison with the ICfree cohort. Healthcare resources utilization was also assessed.

\section{Methods}

\section{Study design, population and setting}

This is a population-based retrospective cohort study using real-world data from the VID, including a population $\geq 18$ years old living in the Valencia Region between 2009 and 2014. The inclusion date was defined as 1st January 2009 for those subjects older than 18 years continuously registered in the RHS for at least 12 months before this date, or first date after 1st January 2009 when the subject was continuously registered in the RHS for at least 12 months before this date and was 18 years old. The date of end of follow-up was defined as end of the study period (31st December 2014) or the date of exit of the RHS (including death), whichever comes sooner.

\section{Real world data: the Valencia healthcare integrated databases (VID)}

We used the following registries from VID [28]: 1) the regional population-based administrative database (SIP), that collects and updates demographic data, health services assignment and usage of the health system; 2) the Ambulatory Care Information System (SIA), that contains medical information for each patient attended in the Primary Care (PC) setting (General Practitioners, GPs, and specialists); 3) the minimum basic data set (MBDS), that collects all diagnosis and procedures from 
hospitalizations; and 4) the Care Provision Management (GAIA), that gathers drug prescriptions and dispensations data using the Anatomical Therapeutic Chemical Classification System (ATC). Both SIA and MBDS used the International Classification of Diseases, Ninth Revision, Clinical Modification coding system (ICD-9-MC) for codification. All these registries can be linked at the individual level through a unique personal identification number [28, 32].

\section{Study cohorts}

The IC-cohort consisted of all eligible individuals with an IC condition-related code registered in SIA or MBDS as follows: HSCT, solid organ transplantation (SOT), haematological neoplasia (HN), solid organ neoplasia (SON), neoplasias overall, HIV, rheumatoid arthritis (RA), systemic lupus erythematosus (SLE), IBD, psoriasis, multiple sclerosis (MS), autoimmune thyroiditis (AT) and immunodeficiency disorders and autoimmune diseases overall (autoimmune) (See supplementary Table 1 for ICD-9-MC codes) [33, 34]. Subjects were considered as IC from the date of the first registry until the end of the follow-up period.

The IC-free cohort consisted of subjects with no IC condition-related code detected during the study period. The start of follow-up for the IC-free cohort was the date of inclusion in the study. The IC-free status of an individual changed during the study period if an ICD code for an IC-related condition appeared in MBDS or SIA databases. In the case of PC or hospitalization IC diagnoses, the beginning of IC status was defined as the date in which IC disease was diagnosed and the subject was considered IC from this date until the end of the follow up period (end of the study period or exit from SIP including death).

\section{Endpoints related to $\mathrm{HZ}$}

HZ-incident cases were considered as the first appearance of a HZ-related ICD-code (053.x) [35] in either SIA or MBDS (in any diagnostic position). Any PC medical contact or visit, or hospital admission related to $\mathrm{HZ}$ was considered as a medical encounter. In order to ensure that $\mathrm{HZ}$ cases were new cases, a period of at least 12 months of registration in RHS was required before the inclusion in the study population.

HZ-recurrent cases were considered when new HZrelated ICD-code appeared at least 6 months after a previous $\mathrm{HZ}$ encounter in the same subject. $\mathrm{HZ}$ case identification using databases and ICD-codes have shown elevated positive predictive value $(92.7 \%$; $95 \%$ CI: $89.1-$ 95.4) [32].

HZ-complications were defined as any HZ-related hospitalization with one of the following ICD-codes at discharge in MBDS: central nervous system (CNS) complications (053.0 and 053.1x) which include PHN (053.12, 053.13 and 053.19), ophthalmic complications (053.2x) and other complications (053.7x and 053.8x).

\section{Statistical analysis}

Socio-demographic characteristics (age, gender, calendar year, urban/rural residence, social exclusion risk, health department and comorbidities (diabetes, COPD, HF and chronic kidney diseases (CKD)) of the study population and IC condition type (for the IC cohort), were summarized using descriptive tables including proportions and frequencies.

$\mathrm{HZ}$ incidence (number of cases per 1000 persons-year) and recurrence (number of cases per 100 persons-year) rates were estimated for IC population, globally and stratified by gender, age groups: $18-29,30-39,40-49$, $50-59,60-69,70-79 \mathrm{y} \geq 80$ years, calendar year and type of IC condition. The respective $95 \%$ confidence intervals (CI) were calculated by the exact method of Poisson.

The risk of HZ, HZ recurrence and HZ complications in the IC-cohort subjects respect to IC-free cohort were estimated by a Poisson (quasi-poisson or negative binomial) regression adjusted by age, gender, calendar year, comorbidities (diabetes, COPD, CKD and HF), and health department.

Healthcare resource utilization among IC and IC-free cohorts was estimated as the number of HZ-related PC visits, number and length of HZ-related hospitalizations, number and duration of sick leaves due to $\mathrm{HZ}$ and prescriptions and dispensations of antivirals (Aciclovir, Famciclovir and Valaciclovir) during the six months following the HZ diagnosis. We performed different statistical General Linear Models (GLM) to compare the populations with and without IC conditions (see supplementary material 2).

\section{Ethical considerations}

The study protocol was approved by the Ethics Committee of Dirección General de Salud Pública / Centro Superior de Investigación en Salud Pública, which allowed for the linkage of the different databases by the administrators database using a codified SIP number.

\section{Results}

\section{Study population}

The study population included 4,382,590 individuals aged $\geq 18$ years that met the inclusion criteria $(49 \%$ male). This cohort included 578,873 (13\% of the study population) individuals with IC (48\% male). Among the IC conditions, neoplasia (7\%) was the most frequent, including SON (5.9\%) and HN (1.3\%). They were followed by psoriasis (3.3\%) and RA (0.9\%). Diabetes was registered in $11.2 \%$ of the population, COPD in $5.1 \%$, CKD in $3.4 \%$ and $\mathrm{HF}$ in $3.2 \%$. Some conditions were more 
frequent among men such as HIV (71.4\%) or COPD (66.2\%) and some other among women including SLE (84.2\%), MS (68.9\%) or RA (68.2\%). Sample size overall and by IC condition in the IC-cohort and IC-free, age distribution and demographic characteristics are listed in Table 1.

\section{$\mathrm{HZ}$ incidence rates}

Incidence rate (IR) (in 1000 persons-year) of HZ (including $\mathrm{PC}$ and hospital registries) was 5.02 in population overall, 9.15 in IC cohort and 4.65 in IC-free cohort. IR of $\mathrm{HZ}$ increased with age, in IC-free cohort the lowest IR was 2.26 in 18-29 age group, and the highest IR was 9.54 in $\geq 80$ age group. In IC cohort the lowest IR was 3.61 in 18-29 age group and the highest was 12.93 in 70-79 age group (Table 2).

IR of $\mathrm{HZ}$ remained almost constant throughout the study period with no significant trend in any of the cohorts (not shown). The overall IR of $\mathrm{HZ}$ was higher in women than in men in both IC (9.74 vs. 8.48 cases/1000 persons-year) and IC-free cohorts (5.43 vs. 3.83 cases/ 1000 persons-year) (Fig. 1).

The overall IR (in 1000 persons-year) of $\mathrm{HZ}$ in the studied IC conditions ranged from 6.13 for psoriasis to 56.07 for HSCT (Table 2). IR for the other IC conditions were: 13.36 for SLE, 12.94 for HIV, 12.65 for SOT, 11.99 for HN, 11.05 for RA, 10.97 for SON, 8.29 for IBD, 7.88 for immunodeficiency disorders and autoimmune diseases, 7.19 for AT and 6.33 for MS (Table 2).

The adjusted risk of $\mathrm{HZ}$ increased by $51 \%$ among people with IC conditions with respect to people IC-free (relative risk [RR] 1.51, 95\% CrI: 1.48-1.54). It was 33\% higher among females than that of males (RR 1.33, 95\% CI: 1.31-1.35) and it increased with age (Table 3). The $\mathrm{HZ}$ risk was $36 \%$ higher for patients with COPD, $12 \%$ for diabetes, $16 \%$ for HF and 18\% for CKD (Table 3).

\section{Incidence rates of $\mathrm{HZ}$ - related complications}

The IR (in 100,000 persons-year) of HZ related complications was 3.63 in population overall, 14.36 in the IC cohort and 2.64 in the IC-free cohort (Table 4). The most common complications were CNS (70.7\%) which mainly included PHN (65\%), followed by ophthalmic complications (15.93\%) and other complications (13.35\%).

IC patients had double risk of hospitalization by a $\mathrm{HZ}$ complication (Table 3). HZ complications risk was 15\% higher in women and increased exponentially with age. The risk was approximately double in COPD (vs. NOCOPD) and HF (vs. No-HF) patients and increased by $7 \%$ in patients with diabetes and by $63 \%$ in CKD population.

\section{Recurrence of $\mathrm{HZ}$}

The recurrence rates (in 100 persons-year) of $\mathrm{HZ}$ were $1.66,2.28$ and 1.56 in the population overall, the IC and the IC-free cohorts, respectively. The highest recurrence rate was found in people with HSCT (5.5/100 personsyear), followed by people with HN (3.35/100 personsyear) and HIV (3.2/100 persons-year) (Table 5).

The adjusted risk of developing a recurrent $\mathrm{HZ}$ increased by $25 \%$ among people with IC conditions respect to people IC-free (RR 1.25, 95\% CrI: 1.19-1.31) (Table 3). The HZ recurrence was $26,5,63$ and $20 \%$ higher for people with COPD, diabetes, HF and CKD, respectively.

\section{Healthcare resources utilization}

Overall, HZ-related healthcare resources utilization was higher in the IC-cohort than those IC-free (Table 6): their HZ-related hospitalizations were three times higher (OR 2.93; 95\% CI: 2.62-3.27) with 12\% longer hospital stays (Mean ratio: 1.12; 95\% CI: 1.02-1.23), their HZspecialized visits were 66\% higher (RR 1.66; 95\% CI: $1.57-1.76)$, and they attended $2 \%$ more frequently to PC (RR 1.02; 95\% CI: 1.01-1.03). In addition, their average of sick leave duration in days was $18 \%$ longer (Mean ratio: 1.18 ; 95\% CI: $1.03-1.35$ ) and the antiviral dispensation was $20 \%$ higher than in IC-free.

\section{Discussion}

This is the first large (>4 million) population-based study to date investigating the incidence of $\mathrm{HZ}$, its complications and healthcare resources utilization in $\geq 18$ years-old adults in Spain with a wide variety of IC conditions [15]. Our study showed that the adjusted risk of $\mathrm{HZ}$ increased by $51 \%$ among people with IC conditions. The overall incidence of $\mathrm{HZ}$ among individuals with the studied IC conditions considering PC visits and hospitalizations was almost twice as high as the incidence in the IC-free cohort. All the selected IC conditions were above the estimated overall IR in the IC-free cohort, rising up to twelvefold in patients with HSCT. The IR of HZ complications was about 5 times as high in the IC as in the IC-free cohorts. All these findings support that the selected IC conditions increase the risk and severity of $\mathrm{HZ}$ episodes and results in higher healthcare resources utilization.

The observed IR of HZ (5.02/1000 persons-year) for the overall study population $(4,382,590$ individuals aged $\geq 18$ years) is aligned with previous publications [3, 15 , 36]. Despite methodological differences such as case definitions or study designs that makes difficult the comparison in absolute values, our findings are consistent with those of other countries studies assessing burden of $\mathrm{HZ}$ disease in subjects with different IC conditions, in which there is a strong effect of impaired immune system on the risk of HZ, being HSCT, SLE, HIV or SOT 


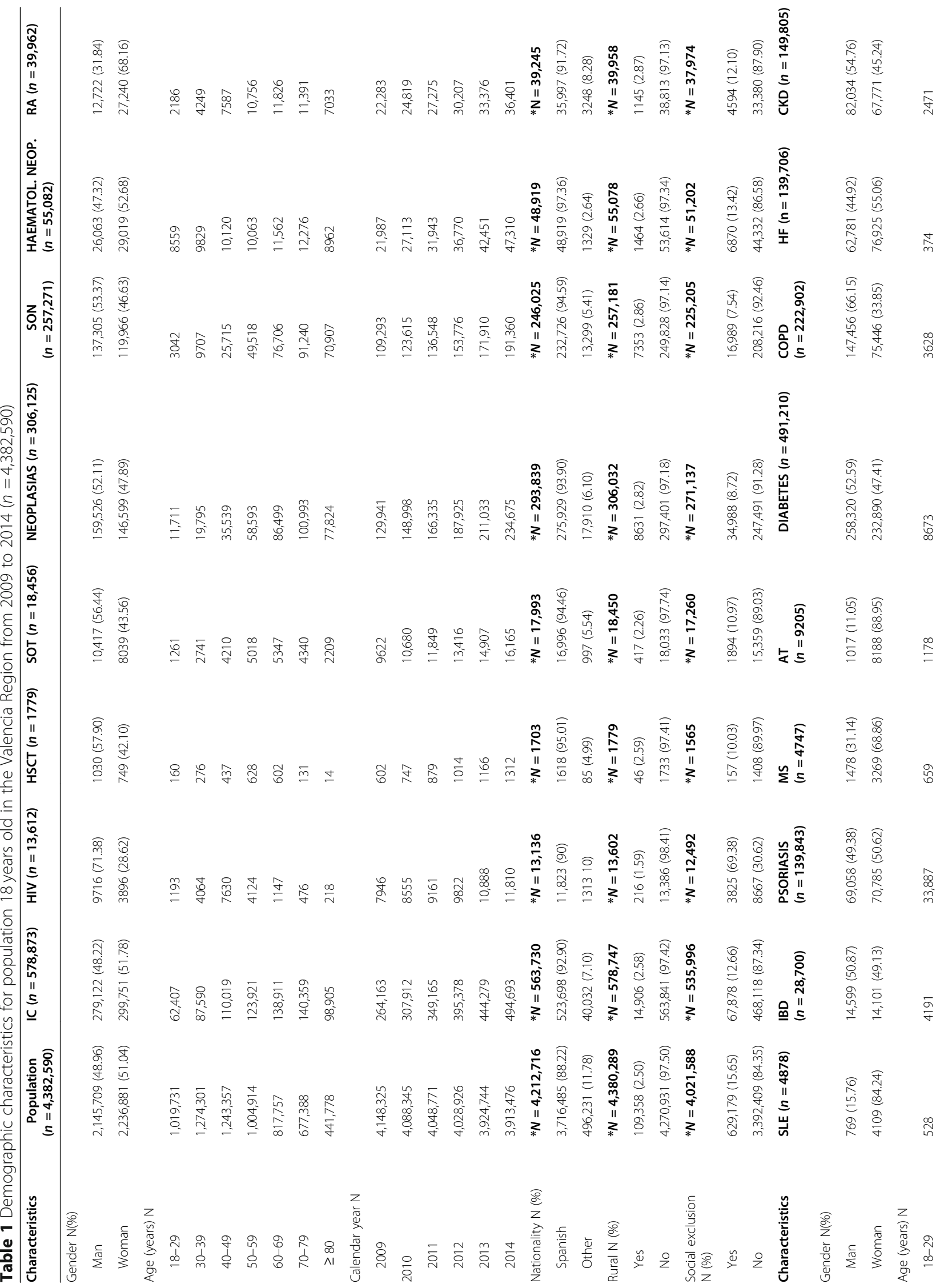


Muñoz-Quiles et al. BMC Infectious Diseases

(2020) 20:905

Page 6 of 14

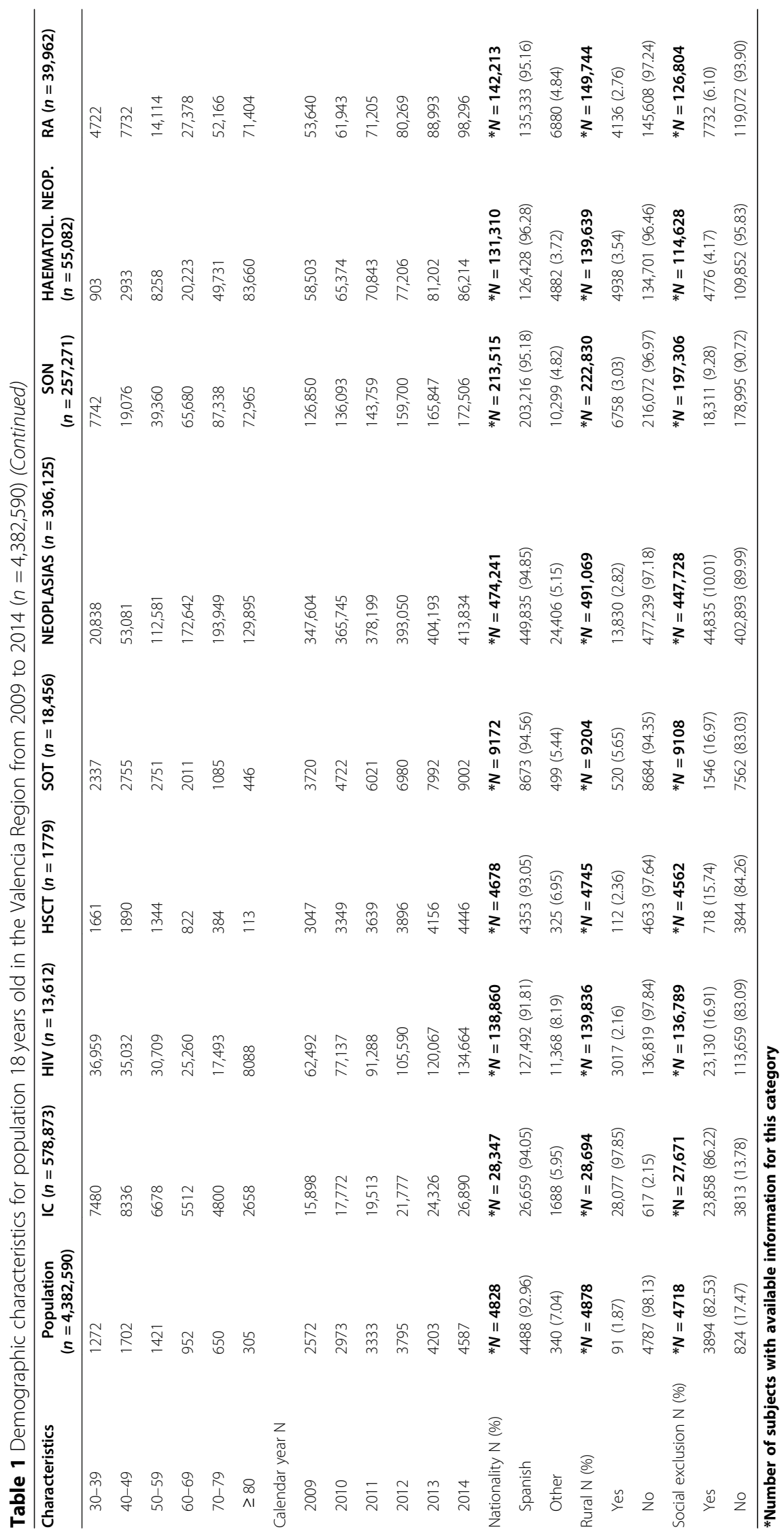




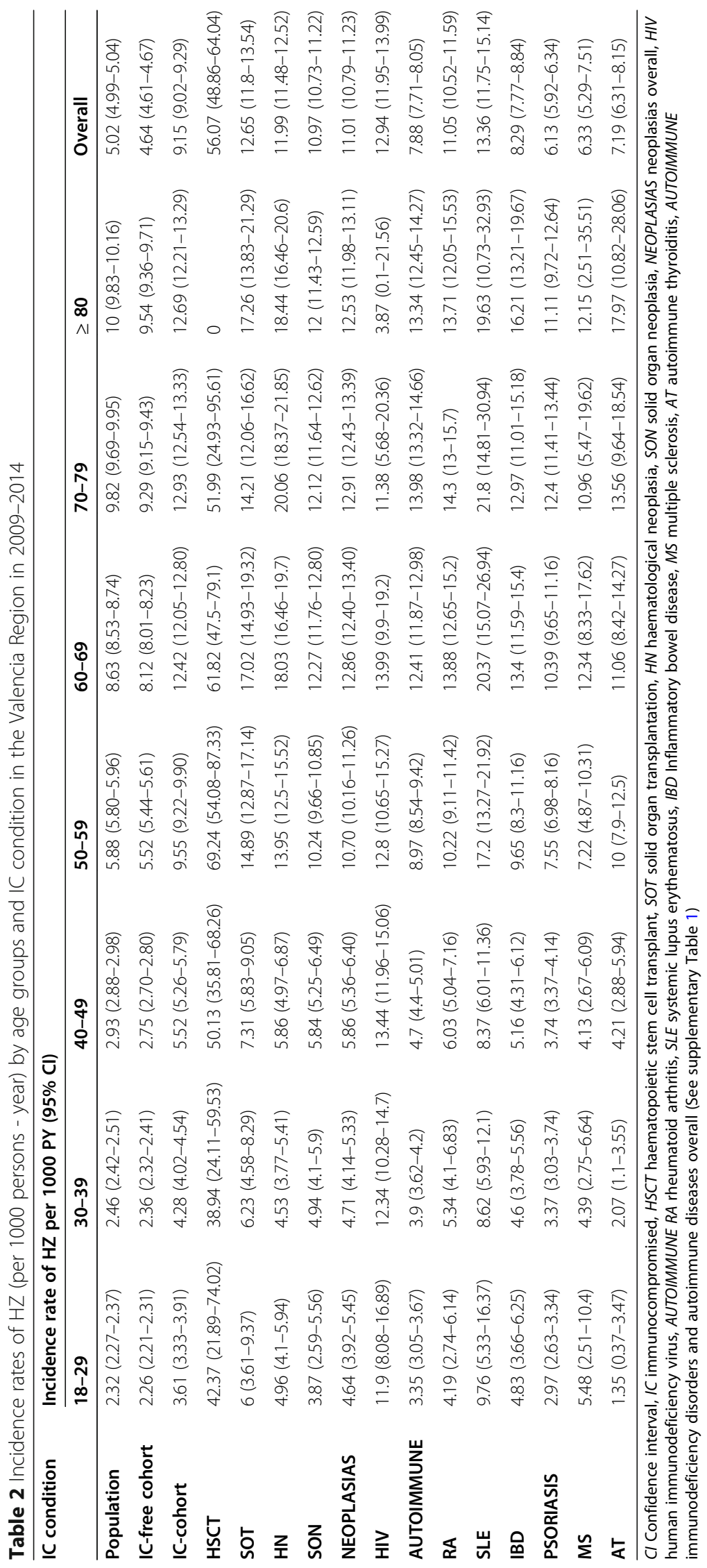




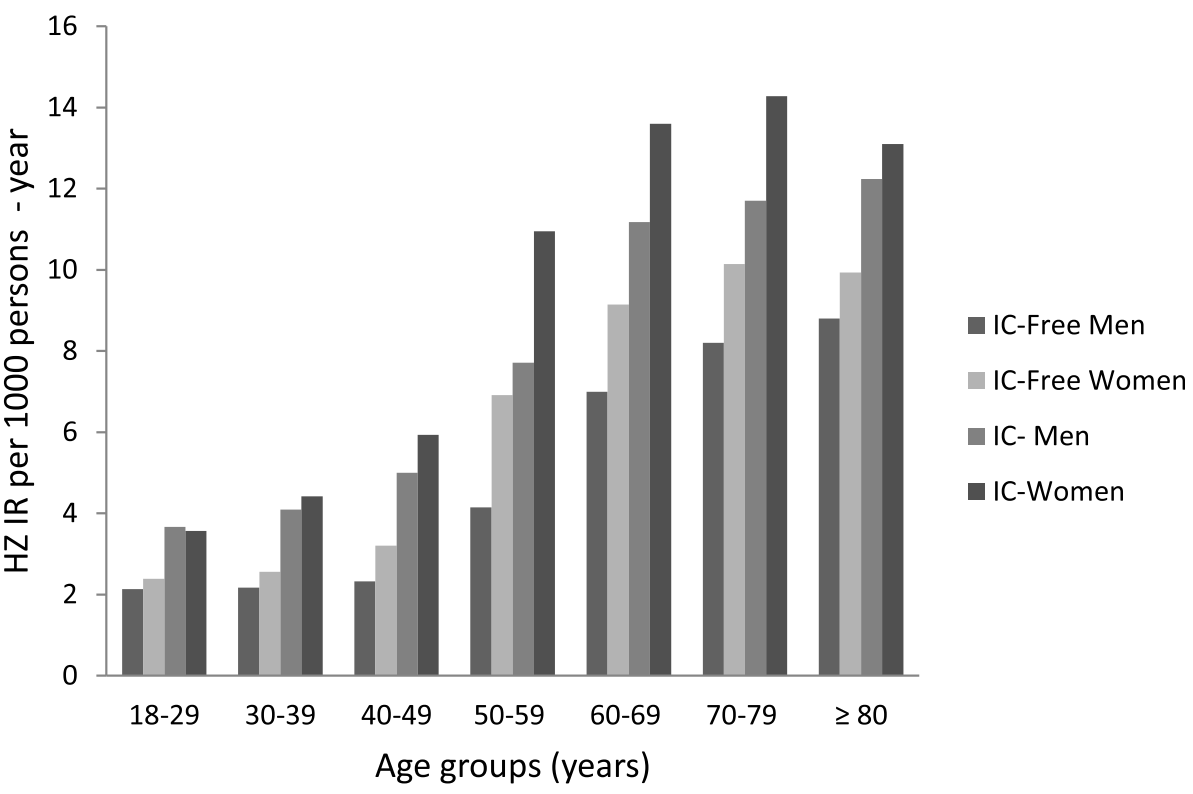

Fig. 1 Incidence rates of $\mathrm{HZ}$ (per 1000 persons-year) in IC and IC-free cohorts by sex and age groups

Table 3 Relative risk (RR) estimates and 95\% credible intervals (95\% Crl) for the association between IC condition and HZ incidence, $\mathrm{HZ}$-complications and $\mathrm{HZ}$ recurrence, controlling by sex, age, comorbidities (COPD, diabetes, HF and CKD), health department (as a random effect) and calendar year, using Bayesian Poisson regression

\begin{tabular}{|c|c|c|c|c|}
\hline & ${ }^{\mathrm{a}} \mathrm{HZ} \mathrm{RR}(95 \% \mathrm{Cr}$ & & $\begin{array}{l}{ }^{\mathrm{b}} \mathrm{HZ}-\text { Complications } \\
\text { RR ( } 95 \% \mathrm{Crl})\end{array}$ & $\begin{array}{l}{ }^{\mathrm{b}} \mathrm{HZ} \text {-Recurrence } \\
\text { RR (95\% Crl) }\end{array}$ \\
\hline \multirow[t]{2}{*}{$\overline{\text { IC }}$} & IC-free & 1 & 1 & 1 \\
\hline & IC & $1.51(1.48-1.54)$ & $2.37(2.01-2.8)$ & $1.25(1.19-1.31)$ \\
\hline \multirow[t]{2}{*}{ SEX } & Man & 1 & 1 & 1 \\
\hline & Woman & $1.33(1.31-1.35)$ & $1.15(0.98-1.35)$ & $1.19(1.14-1.24)$ \\
\hline \multirow[t]{7}{*}{ AGE } & $18-29$ & 1 & 1 & 1 \\
\hline & $30-39$ & $1.06(1.02-1.09)$ & $5.62(2.63-11.99)$ & $1.08(0.95-1.23)$ \\
\hline & $40-49$ & $1.24(1.2-1.29)$ & $9.71(4.9-19.24)$ & $1.14(1.01-1.28)$ \\
\hline & $50-59$ & $2.42(2.35-2.5)$ & $7.74(3.95-15.16)$ & $1.21(1.08-1.36)$ \\
\hline & $60-69$ & $3.43(3.32-3.54)$ & $12.3(6.84-22.1)$ & $1.52(1.36-1.7)$ \\
\hline & $70-79$ & $3.72(3.61-3.85)$ & $26.14(14.72-46.44)$ & $1.9(1.7-2.12)$ \\
\hline & $\geq 80$ & $3.57(3.45-3.7)$ & $39.72(22.5-70.12)$ & $2.14(1.91-2.4)$ \\
\hline \multicolumn{5}{|c|}{ COMORBIDITIES } \\
\hline \multirow[t]{2}{*}{ COPD } & NO-COPD & 1 & 1 & 1 \\
\hline & COPD & $1.36(1.32-1.4)$ & $2.54(2.11-3.06)$ & $1.26(1.17-1.35)$ \\
\hline \multirow[t]{2}{*}{ DIABETES } & NO-DIABETES & 1 & 1 & 1 \\
\hline & DIABETES & $1.12(1.09-1.14)$ & $1.07(0.89-1.28)$ & $1.05(1-1.11)$ \\
\hline \multirow[t]{2}{*}{$\mathrm{HF}$} & NO-HF & 1 & 1 & 1 \\
\hline & $\mathrm{HF}$ & $1.16(1.11-1.21)$ & $2.07(1.68-2.55)$ & $1.63(1.39-1.9)$ \\
\hline \multirow[t]{2}{*}{ CKD } & NO-CKD & 1 & 1 & 1 \\
\hline & CKD & $1.18(1.13-1.23)$ & $1.63(1.31-2.03)$ & $1.2(1.09-1.33)$ \\
\hline
\end{tabular}

${ }^{\mathrm{a}} \mathrm{A}$ Quasi poisson regression model adjusted by sex, age, calendar year, health department and comorbidities (COPD, diabetes, HF and CKD) was used to estimate the risk of $\mathrm{HZ}$ in IC respect to IC-free subjects

${ }^{\mathbf{b}} \mathrm{A}$ Zero-inflated negative binomial model adjusted by sex, age, calendar year, health department and comorbidities (COPD, diabetes, HF and CKD) was used to estimate the risk of $\mathrm{HZ}$ complications (based on hospitalization diagnoses) and recurrence in IC respect to IC-free 


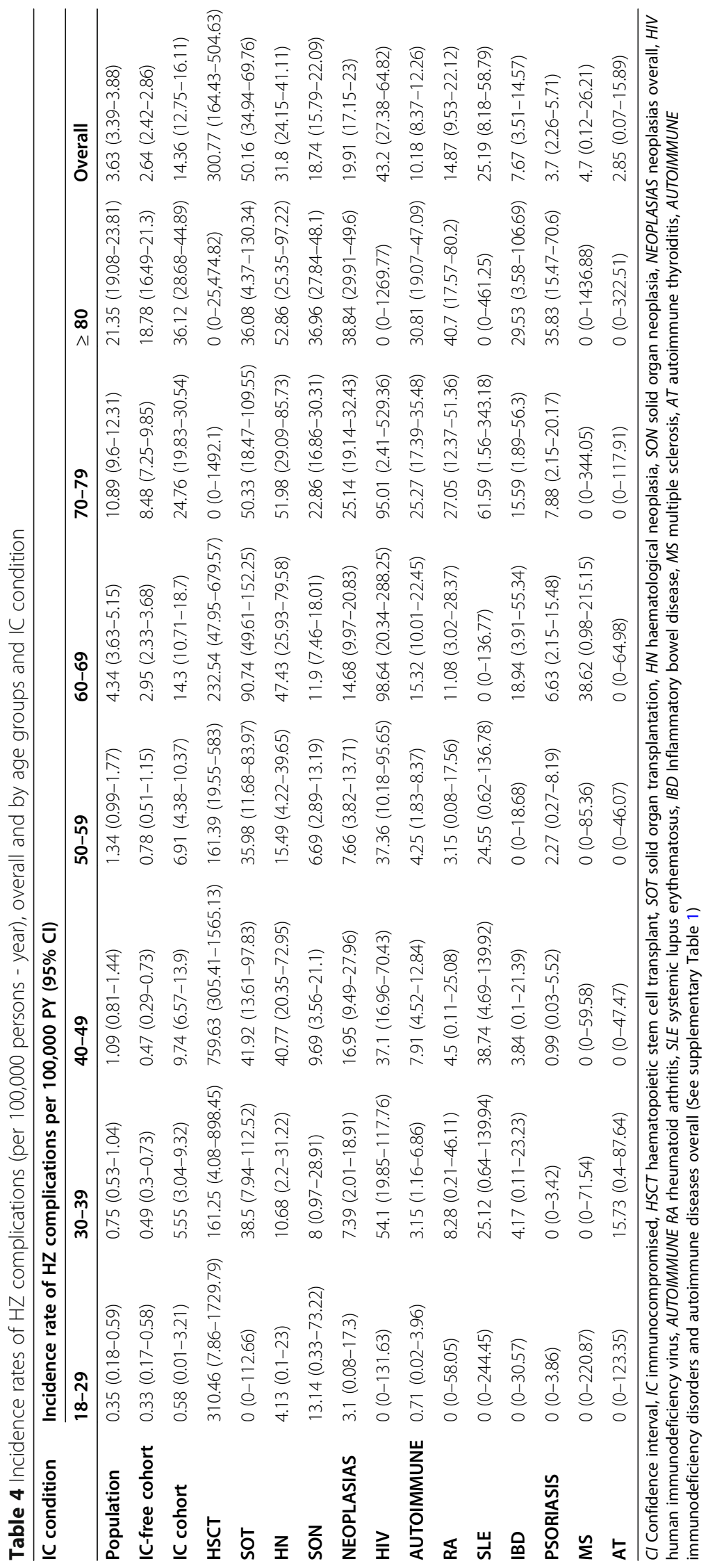




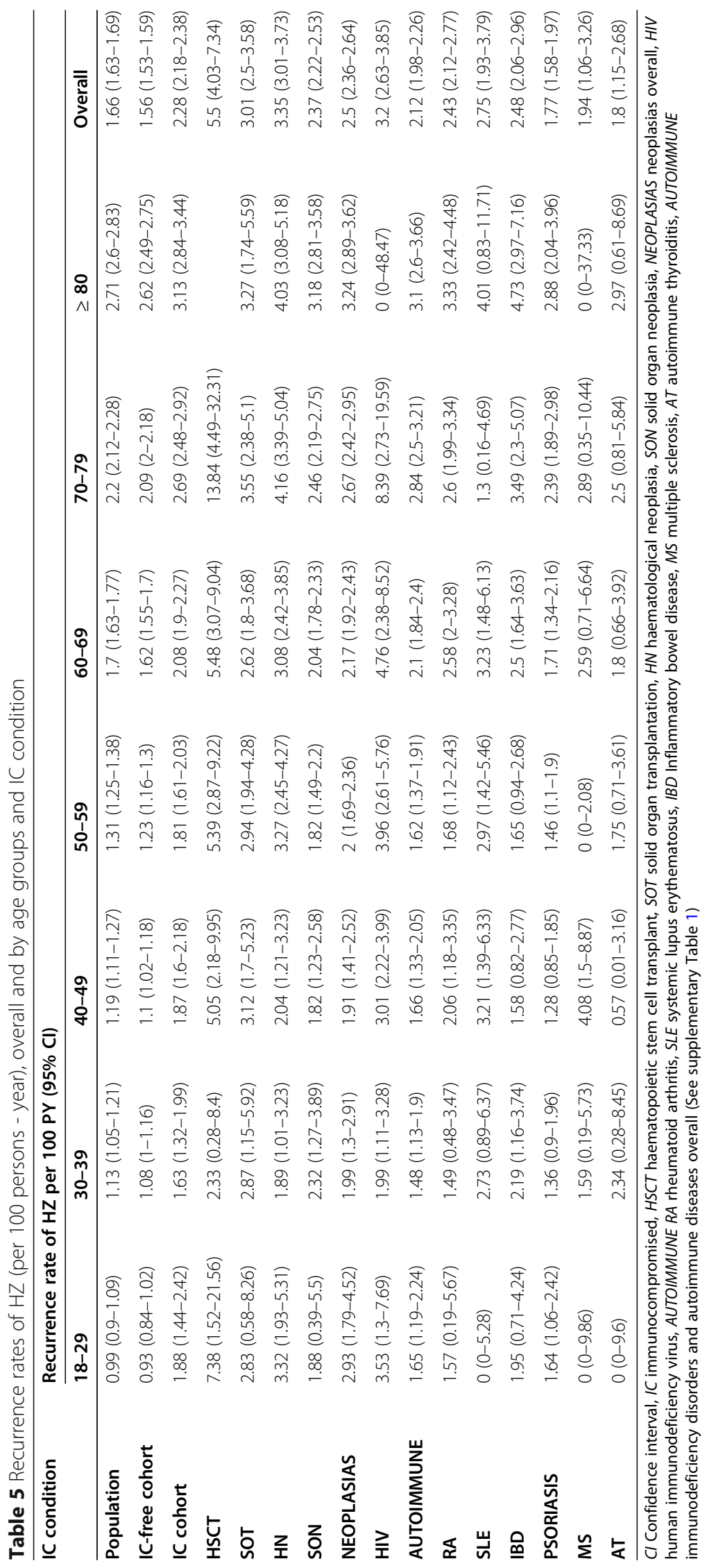


Table 6 HZ- healthcare resources utilization by IC subjects in relation to IC-free

\begin{tabular}{ll}
\hline PC visits for $\mathrm{HZ}$ & IC \\
\hline Specialist visits for $\mathrm{HZ}$ & $\mathrm{RR}(\mathbf{9 5} \% \mathrm{Cl})$ \\
& $1.02(1.01-1.03)$ \\
Hospitalizations $^{\mathrm{a}}$ & $\mathrm{RR}(\mathbf{9 5 \%} \mathrm{Cl})$ \\
& $1.66(1.57-1.76)$ \\
Length of hospital stay & OR (95\% Cl) \\
& $2.93(2.62-3.27)$ \\
Sick leave & Mean ratio $(\mathbf{9 5} \% \mathrm{Cl})$ \\
& $1.12(1.02-1.23)$ \\
Medication for $\mathrm{HZ}$ & Mean ratio (95\% Cl) \\
& $1.18(1.03-1.35)$ \\
\hline
\end{tabular}

aHospitalizations with a $\mathrm{HZ} \mathrm{CIE-9} \mathrm{code} \mathrm{in} \mathrm{any} \mathrm{diagnostic} \mathrm{position;} \mathrm{Cl}$

Confidence interval, $R R$ Relative risk, OR Odds ratio

among the most prone to develop a HZ [5, 26, 27]. Concretely in UK, with a study population of $3,698,346, \mathrm{HZ}$ IRs in IC subpopulations raised up to sevenfold for HSCT patients [26], being the most susceptible to $\mathrm{HZ}$ as in other publications $[3,5,27]$.

In our study, the IC-cohort represented around 13\% of the study population. This percentage is difficult to compare among previous studies from different countries in which the values fluctuate from 1.86 in Japan [27] to 16.8 in UK [26] or 25.5-34 in Germany [5], depending on the selected IC conditions, case definitions, observation period and overall methodological heterogeneity. Nevertheless, aligned with these studies, ours reflects that this IC population involves an excesive proportion of the burden of $\mathrm{HZ}$ in Spain, with an overall risk of $\mathrm{HZ}$ $51 \%$ higher than IC-free population. Interestingly, risks estimations showed in our study were modeled using multivariate models. This is a qualitative difference with previous studies [3, 5, 26, 27] where risks estimations were based on rate comparisons, therefore, they are more prone to bias.

Considering all HZ-related complications, we showed that IC patients have more than twice the risk of hospitalization by a $\mathrm{HZ}$ complication than IC-free people. $70 \%$ of the HZ-related complications were associated to the CNS, with PHN as the most common. In our previous studies we described that $15.7 \%$ of the total $\mathrm{HZ}$ cases developed in people above 50 years old resulted in PHN lasting for at least three months after the acute phase of a HZ $[1,16,17]$. Note that the impact and burden of PHN in those studies could have been underestimated, as subjects with IC conditions were excluded from the study population. This decision was made to avoid confusion, since the only licensed vaccine at the time was the live attenuated vaccine, which is contraindicated for IC population. Despite the differences regarding population and objectives, these studies are complementary and allow us to conclude that HZ complications pose a great threat to IC patients, worsening their health status and raising the amount of health resources required.

Most of the publications agree that the risk of developing an $\mathrm{HZ}$ rises significantly with age, most likely caused by the decreasing cell-mediated immune response to the VZV due to the immunosenescence [32, 37]. This same upward trend is also observed in IC cohort with higher HZ IRs respect to IC-free in all age groups. Interestingly, the IR of HZ in the IC-population aged 50-59 years (9.55 cases/1000 persons-year) was comparable to the one in people aged $80+$ in the IC-free group $(9.54$ cases/ 1000 persons-year), which indicates that patients with IC conditions are prone to develop $\mathrm{HZ}$ at a younger age than individuals IC-free. Age was a variable also associated with an increased risk of suffering $\mathrm{HZ}$ hospitalizations, $\mathrm{HZ}$ complications and $\mathrm{HZ}$ recurrence.

The recurrence rate of $\mathrm{HZ}$ ranged from $<1.5$ to $6.8 \%$ among different studies [36]. Results variations depended on the age, immune status of the study population and, especially, on the follow-up period, being higher in studies with longer follow-up periods [36]. In our study, the Poisson regression analysis showed that the adjusted HZ-recurrence relative risk was 1.25 times higher in patients with IC conditions than in IC-free.

In addition to age and comorbidities, female gender has also shown an increased risk of developing $\mathrm{HZ}$ and suffering recurrences and PHN. This result is also in concordance with previous publications [38-40]. It has been hypothesized that it could be due to differences in responses to latent viral infections, which is supported by the gender difference also reported in the incidence of herpes simplex [39]. However, sex differences regarding care-seeking behaviour could be also influencing these results based on secondary data [5]. A clue pointing in that direction could be the almost but not significant differences between men and women when $\mathrm{HZ}$ related complications were studied in hospitalization registers in the present study, although more research in this regard is required to draw conclusions.

The greatest strength of our study is, without a doubt, the large study population and the VID, a network of electronic health databases where all the information on each subject (demographic, hospital, PC, specialist, drug prescription and dispensation, etc.) can be linked at the patient level. However, some of its limitations are worth mentioning. Firstly, HZ-related hospitalization rates found are higher than other previously published. It probably represents an overestimation as we considered hospitalizations with $\mathrm{HZ}$ diagnoses in any diagnostic 
position and HZ could represent a secondary diagnosis. Secondly, the proportion of IC population increased during the study period. One of the reasons could be related to IC-cohort case definition, since once a diagnosis of a chronic IC condition was registered the patient remained in the IC-cohort group until the end of the follow-up period. It should also be considered that the individuals with none of the selected IC conditions diagnosed could be not necessarily immunocompetent. On the other hand, severity grade of the studied IC conditions and IC-related therapies have not being considered in this study which may impact the HZ IR and its complications. This lack opens the way for new research in this regard looking for associations between the severity or therapy and time to HZ episode.

As we previously have shown for some other underlying conditions such as Diabetes, COPD, or HF $[16,17]$, the greater risk of $\mathrm{HZ}$ in cohorts with IC conditions is linked to an increase in the utilization of health care resources. They attended more frequently to PC and specialized clinics due to $\mathrm{HZ}$, were hospitalized more often and during longer periods, consumed more antivirals and their sick leaves due to $\mathrm{HZ}$ were longer. These data suggest that $\mathrm{HZ}$ episodes were more severe in the ICcohort, contributing to a great reduction in their quality of life and increasing their healthcare-related costs, as previously indicated [41-43].

\section{Conclusion}

In this study we estimate the burden of $\mathrm{HZ}$ disease in people with IC conditions in a Spanish Region of 5 million inhabitants. All the IC conditions studied were above the estimated overall IR in the IC-free cohort and the IR of $\mathrm{HZ}$ complications was about 5 times as high in the IC as in the IC-free cohorts. In conclusion, the selected IC conditions increase the risk and severity of $\mathrm{HZ}$ episodes and result in higher healthcare resources utilization. This first step should be followed by cost effectiveness studies that help us establishing the value of vaccinating these groups of patients when the new prevention strategies become approved in those risk groups.

\section{Supplementary Information}

Supplementary information accompanies this paper at https://doi.org/10. 1186/s12879-020-05648-6.

\section{Abbreviations}

AT: Autoimmune thyroiditis; ATC: Anatomical therapeutic chemical; Cl: Confidence interval; CKD: Chronic kidney diseases; CNS: Central nervous system; COPD: Chronic obstructive pulmonary disease; Crl: Credible interval; GAIA: Care provision management (Sistema de información farmacéutica); GLM: Generalized linear models; HF: Heart failure; HIV: Human immunodeficiency virus; HN: Haematological neoplasia; HSCT: Haematopoietic stem cell transplant; HZ: Herpes zoster;
IBD: Inflammatory bowel disease; IC: Immunocompromised; ICD-9CM: International classification of diseases, Ninth Revision. Clinical modification; IR: Incidence rate; MBDS: Minimum basic data set; MS: Multiple sclerosis; OR: Odds ratio; PC: Primary care; PHN: Post-herpetic neuralgia; RA: Rheumatoid arthritis; ReR: Recurrence rate; RHS: Regional Health System; RR: Relative risk; RWD: Real world data; RZV: Recombinant zoster vaccine; SIA: Ambulatory information system (Sistema de información ambulatoria); SIP: Personal identification system; SLE: Systemic lupus erythematosus; SON: Solid organ neoplasia; SOT: Solid organ transplantation; VID: Valencia healthcare Integrated Databases; ZVL: Zoster vaccine live; VZV: Varicella zoster virus

\section{Acknowledgements}

Not Applicable.

\section{Authors' contributions}

AOS is the guarantor of the paper, taking responsibility for the integrity of the work as a whole, from inception to published article. CMQ contributed to study conception and design; data acquisition, analysis, and interpretation; drafting the article and final approval of the version to be published. CMQ takes responsibility for the integrity of the data and the accuracy of the data analysis and serves as principal author. MLL contributed to data acquisition, data cleaning, analysis and interpretation; drafting the article or revising it critically for important intellectual content; and final approval of the version to be published. CMQ and MLL have equally contributed to this work. AOS and JDD contributed to study design; drafting the article or revising it critically for important intellectual content; and final approval of the version to be published. All authors have read and approved the manuscript.

\section{Funding}

Funding for this study was provided by GlaxoSmithKline Biologicals SA (GSK study identifier 207685). GlaxoSmithKline Biologicals SA was provided the opportunity to review a preliminary version of this manuscript for factual accuracy but the authors are solely responsible for final content and interpretation. The Company (GSK) had no role in the analysis or discussion of the results.

\section{Availability of data and materials}

The datasets generated and/or analysed during the current study are available in:

https://drive.google.com/drive/folders/11 ev5TLPrdJyCDOKgpPuPYdQuZXoj1 Wo1?usp=sharing

\section{Ethics approval and consent to participate}

The study protocol, observational in design and using retrospective anonymized non-identifiable data transferred from the Valencia Ministry of Health to the research team according to the Spanish laws and institutional requirements, was approved by the Ethics Committee of Dirección General de Salud Pública / Centro Superior de Investigación en Salud Pública (CEIC DGSP-CSISP). The Valencia Health System Data Commission [28] granted permission for the transfer of specific anonymized data for the study development. Additionally, being that it is a retrospective study with 6 years of study period (2009-2014), it would have been not possible to get individual informed consent from the whole population studied (more than 4 million inhabitants). Based on Helsinki's Declaration (principle 32) and the Law of Biomedical Reseach in Spain (Art. 58.2), the Ethics Committee accepted the exemption.

\section{Consent for publication}

Not Applicable.

\section{Competing interests}

JDD and his institution received research grants from GSK and SPMSD related to $\mathrm{HZ}$ vaccine. He also acted as advisor for these vaccines to GSK and SPMSD. CMQ, AOS and MLL have attended to several congresses whose registration, travel and accommodation costs have been covered by GSK and SPMSD.

\section{Author details}

${ }^{1}$ Vaccines Research Unit, Fundación para el Fomento de la Investigación Sanitaria y Biomédica de la Comunitat Valenciana, FISABIO-Public Health, 
Avda. Cataluña, 21, 46020 Valencia, Spain. ${ }^{2}$ Universidad Católica de Valencia San Vicente Mártir, Carrer de Quevedo, 2, 46001 València, Spain.

\section{Received: 22 July 2020 Accepted: 22 November 2020} Published online: 30 November 2020

\section{References}

1. Munoz-Quiles C, Lopez-Lacort M, Orrico-Sanchez A, Diez-Domingo J. Impact of postherpetic neuralgia: a six year population-based analysis on people aged 50 years or older. J Infect. 2018;77(2):131-6.

2. Arvin AM. Humoral and cellular immunity to varicella-zoster virus: an overview. J Infect Dis. 2008;197:S58-60..

3. Chen SY, Suaya JA, Li Q, Galindo CM, Misurski D, Burstin S, et al. Incidence of herpes zoster in patients with altered immune function. Infection. 2014; 42(2):325-34.

4. Forbes HJ, Bhaskaran K, Thomas SL, Smeeth L, Clayton T, Langan SM. Quantification of risk factors for herpes zoster: population based casecontrol study. BMJ (Clinical research ed). 2014;348:g2911.

5. Schroeder C, Enders D, Schink T, Riedel O. Incidence of herpes zoster amongst adults varies by severity of immunosuppression. J Infect. 2017; 75(3):207-15.

6. Drolet $M$, Brisson $M$, Schmader KE, Levin MJ, Johnson R, Oxman MN, et al. The impact of herpes zoster and postherpetic neuralgia on health-related quality of life: a prospective study. CMAJ : Canadian Medical Association journal = journal de l'Association medicale canadienne. 2010;182(16):1731-6.

7. Gater A, Uhart M, McCool R, Preaud E. The humanistic, economic and societal burden of Herpes Zoster in Europe: a critical review. BMC public health. 2015:15:1514.

8. Johnson RW, Rice ASC. Postherpetic neuralgia. N Engl J Med. 2014;371(16): 1526-33.

9. Forbes HJ, Bhaskaran K, Thomas SL, Smeeth L, Clayton T, Mansfield K, et al. Quantification of risk factors for postherpetic neuralgia in herpes zoster patients: a cohort study. Neurology. 2016;87(1):94-102.

10. Chen N, Li Q, Yang J, Zhou M, Zhou D, He L. Antiviral treatment for preventing postherpetic neuralgia. Cochrane Database Syst Rev. 2014 Feb 6; 2:CD006866. https://doi.org/10.1002/14651858.CD006866.pub3.

11. Johnson RW, Alvarez-Pasquin M-J, Bijl M, Franco E, Gaillat J, Clara JG, et al. Herpes zoster epidemiology, management, and disease and economic burden in Europe: a multidisciplinary perspective. Therapeutic Advances Vaccines. 2015;3(4):109-20.

12. Oxman MN, Levin MJ, Johnson GR, Schmader KE, Straus SE, Gelb LD, et al. A vaccine to prevent herpes zoster and postherpetic neuralgia in older adults. N Engl J Med. 2005;352(22):2271-84.

13. Harpaz R, Ortega-Sanchez IR, Seward JF. Advisory Committee on Immunization Practices Centers for D, Control and P. Prevention of herpes zoster: recommendations of the Advisory Committee on Immunization Practices (ACIP). MMWR Recommendations Rep. 2008;57(RR-5):1-30 quiz CE2-4. PMID: 18528318.

14. Izurieta HS, Wernecke M, Kelman J, Wong S, Forshee R, Pratt D, et al. Effectiveness and duration of protection provided by the live-attenuated herpes zoster vaccine in the Medicare population ages 65 years and older Clin Infect Dis. 2017;64(6):785-93.

15. Mareque M, Oyaguez I, Morano R, Casado MA. Systematic review of the evidence on the epidemiology of herpes zoster: incidence inthegeneral population and specific subpopulationsin Spain. Public Health. 2019;167: $136-46$

16. Muñoz-Quiles C, López-Lacort M, Díez-Domingo J. Risk and impact of herpes zoster among COPD patients: a population-based study, 2009-2014. BMC Infect Dis. 2018;18(1):203.

17. Muñoz-Quiles C, López-Lacort M, Ampudia-Blasco FJ, Díez-Domingo J. Risk and impact of herpes zoster on patients with diabetes: a population-based study, 2009-2014. Hum Vaccin Immunother. 2017:i13(11):2606-2611. https:// doi.org/10.1080/21645515.2017.1368600.

18. Forbes HJ, Thomas SL, Smeeth L, Clayton T, Farmer R, Bhaskaran K, et al. A systematic review and meta-analysis of risk factors for postherpetic neuralgia. Pain. 2016;157(1):30-54.

19. Varghese L, Curran D, Bunge E, Vroling H, van Kessel F, Guignard A, et al. Contraindication of live vaccines in immunocompromised patients: an estimate of the number of affected people in the USA and the UK. Public Health. 2017;142:46-9.
20. Lal H, Cunningham AL, Godeaux O, Chlibek R, Diez-Domingo J, Hwang S-J, et al. Efficacy of an Adjuvanted herpes zoster subunit vaccine in older adults. N Engl J Med. 2015;372(22):2087-96.

21. Lecrenier N, Beukelaers P, Colindres R, Curran D, De Kesel C, De Saegher J-P, et al. Development of adjuvanted recombinant zoster vaccine and its implications for shingles prevention. Expert Review of Vaccines. 2018;17(7): 619-34.

22. Bastidas A, de la Serna J, El Idrissi M. Effect of recombinant zoster vaccine on incidence of herpes zoster after autologous stem cell transplantation: a randomized clinical trial. Jama-J Am Med Assoc (vol 322, pg 123, 2019). doi: https://doi.org/10.1001/jama.2019.9053.

23. Vink P, Delgado Mingorance I, Maximiano Alonso C, Rubio-Viqueira B, Jung $\mathrm{KH}$, Rodriguez Moreno JF, et al. Immunogenicity and safety of the adjuvanted recombinant zoster vaccine in patients with solid tumors, vaccinated before or during chemotherapy: a randomized trial. Cancer. 2019;125(8):1301-12. https://doi.org/10.1002/cncr.31909.

24. Vink P, Ramon Torrell JM, Sanchez Fructuoso A, Kim S-J, Kim S-I, Zaltzman J, et al. Immunogenicity and safety of the Adjuvanted recombinant zoster vaccine in chronically immunosuppressed adults following renal transplant: a phase 3. Randomized Clin Trial Clin Infect Dis. 2020;70(2):181-90. https:// doi.org/10.1093/cid/ciz177.

25. Satyam VR, Li P-H, Reich J, Qazi T, Noronha A, Wasan SK, et al. Safety of Recombinant Zoster Vaccine in Patients with Inflammatory Bowel Disease. Digestive Diseases Sci. 2020;65(10):2986-91. https://doi.org/10.1007/s10620019-06016-4.

26. Yanni EA, Ferreira G, Guennec M, El Hahi Y, El Ghachi A, Haguinet F, et al. Burden of herpes zoster in 16 selected immunocompromised populations in England: a cohort study in the Clinical Practice Research Datalink 20002012. Bmj Open. 2018;8(6):e020528. https://doi.org/10.1136/bmjopen-2017020528

27. Imafuku S, Matsuki T, Mizukami A, Goto Y, de Souza S, Jegou C, et al. Burden of herpes zoster in the Japanese population with Immunocompromised/chronic disease conditions: results from a cohort study claims database from 2005-2014. Dermatol Ther. 2018. https://doi.org/ 10.1007/s13555-018-0268-8.

28. Garcia-Sempere A, Orrico-Sanchez A, Munoz-Quiles C, Hurtado I, Peiro S, Sanfelix-Gimeno G, et al. Data resource profile: the Valencia health system integrated database (VID). Int J Epidemiol. 2020;49(3):740-41e. https://doi. org/10.1093/ije/dyz266.

29. Lopez-Lacort M, Collado S, Diez-Gandia A, Diez-Domingo J. Rotavirus, vaccine failure or diagnostic error? Vaccine. 2016;34(48):5912-5.

30. Perez-Vilar S, Diez-Domingo J, Lopez-Lacort M, Martinez-Ubeda S, MartinezBeneito MA. Effectiveness of rotavirus vaccines, licensed but not funded, against rotavirus hospitalizations in the Valencia region. Spain Bmc Infectious Diseases. 2015;;65(5):840-850. https://doi.org/10.1093/cid/cix369.

31. Garcia S, Martinez-Cengotitabengoa M, Lopez-Zurbano S, Zorrilla I, Lopez P, Vieta $\mathrm{E}$, et al. Adherence to antipsychotic medication in bipolar disorder and schizophrenic patients a systematic review. J Clin Psychopharmacol. 2016; 36(4):355-71.

32. Morant-Talamante N, Diez-Domingo J, Martinez-Ubeda S, Puig-Barbera J, Aleman-Sanchez S, Perez-Breva L. Herpes zoster surveillance using electronic databases in the Valencian community (Spain). BMC Infect Dis. 2013;13:463.

33. Guignard AP, Greenberg M, Lu C, Rosillon D, Vannappagari V. Risk of herpes zoster among diabetics: a matched cohort study in a US insurance claim database before introduction of vaccination, 1997-2006. Infection. 2014; 42(4):729-35.

34. Suaya JA, Chen S-Y, Li Q, Burstin SJ, Levin MJ. Incidence of herpes zoster and persistent post-zoster pain in adults with or without diabetes in the United States. Open Forum Infect Dis. 2014;1(2):ofu049.

35. Weinmann S, Vandermeer M, Roberts M, Mullooly J, Chun C. Positive predictive value of ICD-9 code for herpes zoster among children during the varicella vaccine era. Pediatr Infect Dis J. 2016;35(4):459-60.

36. Kawai K, Gebremeskel BG, Acosta CJ. Systematic review of incidence and complications of herpes zoster: towards a global perspective. Bmj Open. 2014;4(6):e004833. https://doi.org/10.1136/bmjopen-2014-004833.

37. Schmader K. Herpes zoster in older adults. Clin Infect Dis. 2001:32(10): 1481-6.

38. Opstelten W, Van Essen GA, Schellevis F, Verheij TJ, Moons KG. Gender as an independent risk factor for herpes zoster: a population-based prospective study. Ann Epidemiol. 2006;16(9):692-5. 
39. Fleming DM, Cross KW, Cobb WA, Chapman RS. Gender difference in the incidence of shingles. Epidemiol Infect. 2004;132(1):1-5.

40. Munoz-Quiles C, Lopez-Lacort M, Orrico-Sanchez A, Diez-Domingo J. Letter to the Editor regarding "The role of age-sex interaction in the development of post-herpetic neuralgia". Human vaccines Immunotherap. 2017;14(4):9068. https://doi.org/10.1080/21645515.2017.1417715.

41. Cebrian-Cuenca AM, Diez-Domingo J, San-Martin-Rodriguez M, Puig-Barbera J, Navarro-Perez J, Herpes Zoster Res Grp V. Epidemiology and cost of herpes zoster and postherpetic neuralgia among patients treated in primary care centres in the valencian community of Spain. Bmc Infect Dis. 2011;11: 302. https://doi.org/10.1186/1471-2334-11-302.

42. Meyers JL, Candrilli SD, Rausch DA, Yan S, Patterson BJ, Levin MJ. Cost of herpes zoster and herpes zoster-related complications among immunocompromised individuals. Vaccine. 2018;36(45):6810-8.

43. Torcel-Pagnon L, Bricout H, Bertrand I, Perinetti E, Franco E, Gabutti G, et al. Impact of underlying conditions on zoster-related pain and on quality of life following zoster. J Gerontol Series A-Biol Sci Med Sci. 2017;72(8):1091-7. https://doi.org/10.1093/gerona/glw189.

\section{Publisher's Note}

Springer Nature remains neutral with regard to jurisdictional claims in published maps and institutional affiliations.

- fast, convenient online submission

- thorough peer review by experienced researchers in your field

- rapid publication on acceptance

- support for research data, including large and complex data types

- gold Open Access which fosters wider collaboration and increased citations

- maximum visibility for your research: over $100 \mathrm{M}$ website views per year

At BMC, research is always in progress.

Learn more biomedcentral.com/submissions 